\title{
Statistical Interpretation of Joint Multiplicity Distributions of Neutrons and Charged-particles
}

\author{
J. Tõke, D.K. Agnihotri, W. Skulski, and W.U. Schröder \\ Department of Chemistry and Nuclear Science Research Laboratory, \\ University of Rochester, Rochester, New York 14627
}

\begin{abstract}
Experimental joint multiplicity distributions of neutrons and charged particles provide a striking signal of the characteristic decay processes of nuclear systems following energetic nuclear reactions. They present, therefore, a valuable tool for testing theoretical models for such decay processes. The power of this experimental tool is demonstrated by a comparison of an experimental joint multiplicity distribution to the predictions of different theoretical models of statistical decay of excited nuclear systems. It is shown that, while generally phase-space based models offer a quantitative description of the observed correlation pattern of such an experimental multiplicity distribution, some models of nuclear multifragmentation fail to account for salient features of the observed correlation.
\end{abstract}

PACS numbers: 25.70.-z,25.70.Lm,25.70.Pq 
Nuclear multifragmentation 1 8, the production of multiple intermediate-mass fragments (IMFs) in individual reaction events, has been one of the central issues in intermediateenergy heavy-ion reactions in the last decade. The theoretical effort 1 to understand this phenomenon is largely driven by an expectation that this process may reveal a macroscopic behavior of nuclear matter at very high excitation energies that is qualitatively different from its behavior at lower excitation energies. Of particular interest in this context is the prospect of probing the nuclear liquid-gas phase transition, which has often been associated with multiple IMF production. Parallel with purely theoretical effort, a search has been conducted for experimental signatures that could be connected to certain IMF production scenarios by means of simple and reliable simulation calculations. 11 At the present stage of this research, select partial sets of experimental observations are apparently consistent with different reaction scenarios and mutually exclusive physical concepts. For further progress in the understanding of the reaction mechanism, it is therefore essential to identify reliable experimental observations that would challenge some but not other models and propositions. It now appears, that the directly measured joint distribution of neutron and charged-particle multiplicities is one such reliable observable. The significance of this observable, in general, and for the ongoing discussion regarding the character of multi-fragmentation 9 - 5 , in particular, has gone so far largely unnoticed, due to a seemingly trivial character of the information contained in the above joint distributions. The present work demonstrates the discriminative power of the combined multiplicity observable in two sample analyses.

Fig. 1 shows a typical joint distribution of neutron and light charged-particle multiplicities, $m_{n}$ and $m_{L C P}$, as observed in the ${ }^{209} \mathrm{Bi}+{ }^{136} \mathrm{Xe}$ reaction at $\mathrm{E} / \mathrm{A}=28 \mathrm{MeV}$ and reported on earlier.16 17 The notable feature of the observed distribution is the presence of a welldefined correlation ridge with a characteristic bend around $\left(m_{n}, m_{L C P}\right) \approx(15,0)$. When following the crest of the correlation ridge in Fig. 1 beginning from the origin of the plot, one first encounters a rather long segment running straight and parallel to the $m_{n}$ axis. Along this first segment, $m_{L C P} \approx 0$ and, hence, the role of the charged-particle emission is insignificant. The latter decay mode becomes a factor only when the measured neutron 
multiplicity exceeds rather significant values of the order of $m_{n}=15$ (corresponding to a true, efficiency-corrected value of $m_{n} \approx 30$ ). At above multiplicities of $m_{n} \approx 15$, the crest of the correlation ridge is seen to part with the neutron multiplicity axis and run at a constant angle with respect to the $m_{n}$ axis, until a saturation in both, neutron and LCP multiplicities is reached. It is the presence of the first, $m_{L C P} \approx 0$ segment of the joint distribution and its appreciable length that poses a challenge to some propositions regarding IMF production.

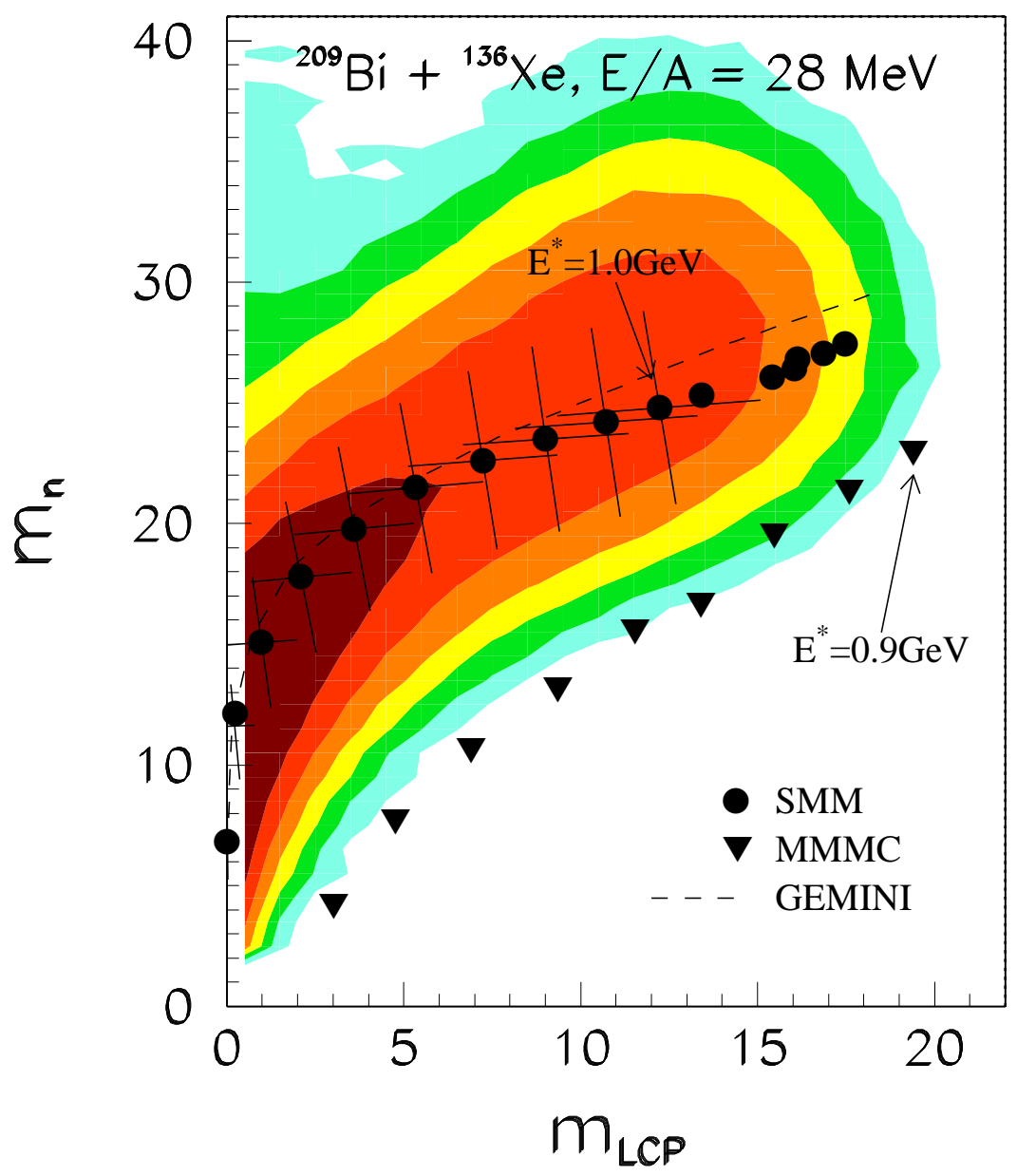

Fig. 1. Experimental ${ }^{9}$ logarithmic (base 2) contour plot of the joint distribution of neutron and charged-particle multiplicities. Trends predicted by the codes $\mathrm{SMM},{ }^{7} \mathrm{MMMC},{ }^{8}$, and GEMINI $^{20}$ are illustrated by circles, squares, and a dashed line, respectively. The slanted bars attached to some of the theoretical data points illustrate the (efficiency-corrected) local orientation and lengths (FWHM) of the major and minor axes of the $m_{n}-m_{L C P}$ covariance tensor for a fixed total excitation energy. 
It is important to emphasize that the above two-segment topography of the correlation ridge in the joint $m_{n}$ vs. $m_{L C P}$ distribution has been observed for many systems and at different bombarding energies. The pertinent feature discussed above is also consistent with earlier observation $\$ 18$ of average neutron and charged-particle multiplicity correlations for other systems. As a matter of fact, the same characteristic shape has been observed in any experiment where such joint distributions or average correlations were measured. These experiments include those studying reactions induced by relativistic protons and antiprotons 19

The behavior seen in Fig. 1 is consistent with a dominantly statistical, phase-space governed, emission scenario of neutrons and light charged products. One expects that the lack of a Coulomb barrier for neutrons would lead to a strong dominance of the neutron emission channels at low excitation energies and, hence, to the presence of the first, $m_{L C P} \approx 0$ segment of the correlation ridge. Simulation calculations confirm 16 that phase-space models, such as the evaporation model20 GEMINI and the Copenhagen modell of simultaneous multifragmentation (SMM), reproduce quite accurately the topography of the correlation ridge in Fig. 1 and, especially, the location of the crest line of the experimental ridge. As an example, the results of SMM7 and GEMINI2 calculations are indicated in Fig. 1 by solid dots and the dashed line, respectively. The "error" bars attached to the dots illustrate the orientation and the lengths (in terms of FWHM) of the main axes of the theoretical correlation tensor of the neutron and LCP multiplicities for fixed excitation energies. In the above model calculations, it was assumed that neutrons and light-charged particles are emitted from fully-accelerated projectile- and target-like fragments produced in the primary dissipative collision. Such an assumption is justified by the experimental observation 23 that, for heavy-ion reactions of interest here and low kinetic-energy losses, particle emission patterns are characteristic of dominantly statistical emission from two equilibrated sources, with only a weak contribution of pre-equilibrium emission to the particle yield. Subsequently, the raw theoretical predictions have been corrected for the efficiency of the Rochester RedBall neutron multiplicity meter, 21 used in the measurement of the joint multiplicity distribution shown in Fig. 1. These efficiencies were calculated using a modified version of the Monte 
Carlo code DENIS,22 which has been calibrated using various sets of experimental data.21 For the assumed binary kinematics, they are in the range of 55\%-61\%.

In view of the above, an interpretation of the correlation ridge seen in Fig. 1 in terms of a dominantly statistical process is well warranted. This is especially true for low total excitation energies, on which the conclusions of the present paper rely.

The importance of the experimental measurements of joint distributions of neutron and LCP multiplicities is demonstrated by the fact that not all prominent models for the decay of hot nuclear matter account for the gross trends observed in the experimental data. Quite obviously then and, possibly, contrary to one's intuition, these trends are not trivial at all. Notably, the Berlin microcanonical fragmentation model, as implemented in the Metropolis Monte Carlo code 8 MMMC, does not reproduce satisfactorily the first, relatively long segment of the $m_{n}$ vs. $m_{L C P}$ correlation ridge parallel to the $m_{n}$ axis. Instead, as illustrated by the triangles in Fig. 1, the model predicts a correlation ridge that is characterized by an approximately straight proportionality between average multiplicities of neutrons and charged-particles. We note, that calculations for all three models were performed for identical initial conditions, to provide for a meaningful comparison.

It is clear from Fig. 1 that MMMC calculations largely underestimate the role of neutron emission channels in the decay of excited nuclear systems and apportion the thermal energy incorrectly to the different particle types. While the physics captured by the MMMC, on the one hand, and the SMM, on the other hand, appears to be very similar, these two codes differ strongly in the way they treat fragment excitation and neutron emission. In the SMM, neutrons are allowed to be emitted sequentially from thermally equilibrated excited primary fragments, whereas the MMMC requires all the emitted neutrons to be in a narrow band of continuum states simultaneously at one particular moment in time, while the core fragments are essentially cold. Obviously, the latter process is statistically much less likely than sequential emission from hot fragments. It is this difference in the treatment of neutron emission that makes the predictions by the MMMC for the $m_{n} v s$. $m_{L C P}$ correlations qualitatively different from the predictions by the SMM for the same 
experimental observable. Furthermore, in the MMMC, the neutron multiplicity is largely determined by the choice of weight factors describing different multiplicities of "evaporated" neutrons. Conceptually, these weight factors are to represent the density of micro states of the collection of all "evaporated" neutrons at a given total energy of this collection. However, in the MMMC, they are approximated by

$W_{p} \propto V_{p}^{m_{e v}}$,

where $V_{p}$ is the volume of a spherical shell in momentum space, enclosed between $p_{\min }=$ $\sqrt{2 U_{n}}\left(U_{n}=50 \mathrm{MeV}\right.$ being the depth of the single-neutron potential well) and a "suitably chosen" $p_{\max }$. In Eq. 1, $m_{e v}$ is the multiplicity of "evaporated" neutrons. The momentum distribution described by Eq. 1, corresponds not to a definite energy but to a broad spectrum of the total energy of all "evaporated" neutrons and, hence, $W_{p}$ are not microcanonical, as are most (but not all remaining) weight factors in the MMMC. One notes, that in the MMMC, the competition by channels with various neutron multiplicities is largely decided by what is termed a "suitable choice" of a critical parameter $p_{\max }$ in a particular (nonmicrocanonical) parameterization of $W_{p}$ via Eq. [1. Therefore, the discrepancy between the MMMC prediction, on the one hand, and the predictions by the codes SMM and GEMINI, on the other hand, cannot be traced to any physical effects, but rather to the accuracy of mathematical/physical shortcuts adopted by the MMMC.

The utility of the joint multiplicity distributions $\left(m_{n}\right.$ vs. $\left.m_{L C P}\right)$ as a powerful tool for scrutinizing theoretical concepts is further demonstrated in the context of pseudo-Arrhenius plots for multi-IMF distributions. In an ongoing discussion, the merits of a proposed statistical interpretation of pseudo-Arrhenius plots for nuclear multi-fragmentation have been

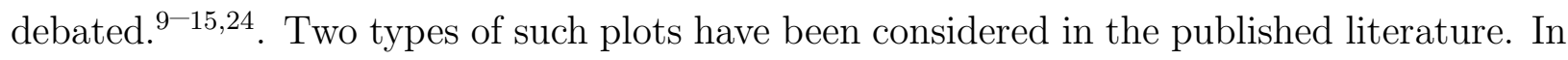
the first one, 10 the logarithm of an inverse "binomial probability" $\ln (1 / p)$ is plotted versus the inverse square root of the transverse kinetic energy $1 / \sqrt{E_{t}}$ of charged reaction products. In the second realization, 1 the average IMF multiplicity $\left\langle m_{I M F}>\right.$ is plotted versus $1 / \sqrt{E_{t}}$. The "binomial probability" $p$ has been introduced $\$$ based on an experimental discovery 
that IMF multiplicity distributions for any given transverse energy are well approximated by binomial distributions:

$$
P_{n}^{m}(p)=\frac{m !}{n !(m-n) !} p^{n}(1-p)^{m-n}
$$

where $m$ and $p$ are the number of trials and the probability for success in any one of these trials, respectively. The transverse energy is defined as $E_{t}=\Sigma E_{k} \sin ^{2}\left(\Theta_{k}\right)$, where the summation extends over all charged products, excluding fission fragments and projectileor target-like residues, and $\Theta_{k}$ is the emission angle of the $k$-th product. The declared, rationale 19,11 for these kinds of plots is the expectation that for statistical IMF emission, the emission probability should be proportional to the Boltzmann factor $e^{-B / T}$, where $B$ is an effective emission barrier and $T$ is the nuclear temperature. This rationale relies critically on the validity of the assumption that the nuclear temperature $T$ is proportional to $\sqrt{E_{t}}$, which presumes a direct proportionality between $E_{t}$ and the thermal excitation energy $E^{*}$. These assumptions also entail that $E^{*}$ is proportional to the square of the temperature, as is proper for a Fermi gas. The validity of the two above assumptions determines the appropriateness of an interpretation of pseudo-Arrhenius plots in terms of thermal scaling, proposed in a series of recent papers $10-13,24$. For example, had $E_{t}$ depended quadratically or exponentially on $E^{*}$, there would have been no obvious justification for choosing an abscissa variable $1 / \sqrt{E_{t}}$ for the pseudo-Arrhenius plots. Accordingly, these papers rely on limited simulation calculations, which always explicitly state and take for granted, a strict proportionality between $E_{t}$ and $E^{*}$. However, the character of the joint multiplicity distributions discussed above demonstrates unambiguously the lack of even an approximate, or average, proportionality between $E_{t}$ and $E^{*}$. This is so, because neutrons are not included in the experimental definition of the transverse energy $E_{t}$ in the quoted work, whereas neutron emission is seen and understood to be essentially the only significant decay channel available to medium-weight or heavy nuclear systems at excitation energies of up to several hundred $\mathrm{MeV}$. As a result, $E_{t} \approx 0$ for the first few hundreds of $\mathrm{MeV}$ of the total excitation energy, precluding direct proportionality between $E_{t}$ and $E^{*}$ at higher 
excitation energies.

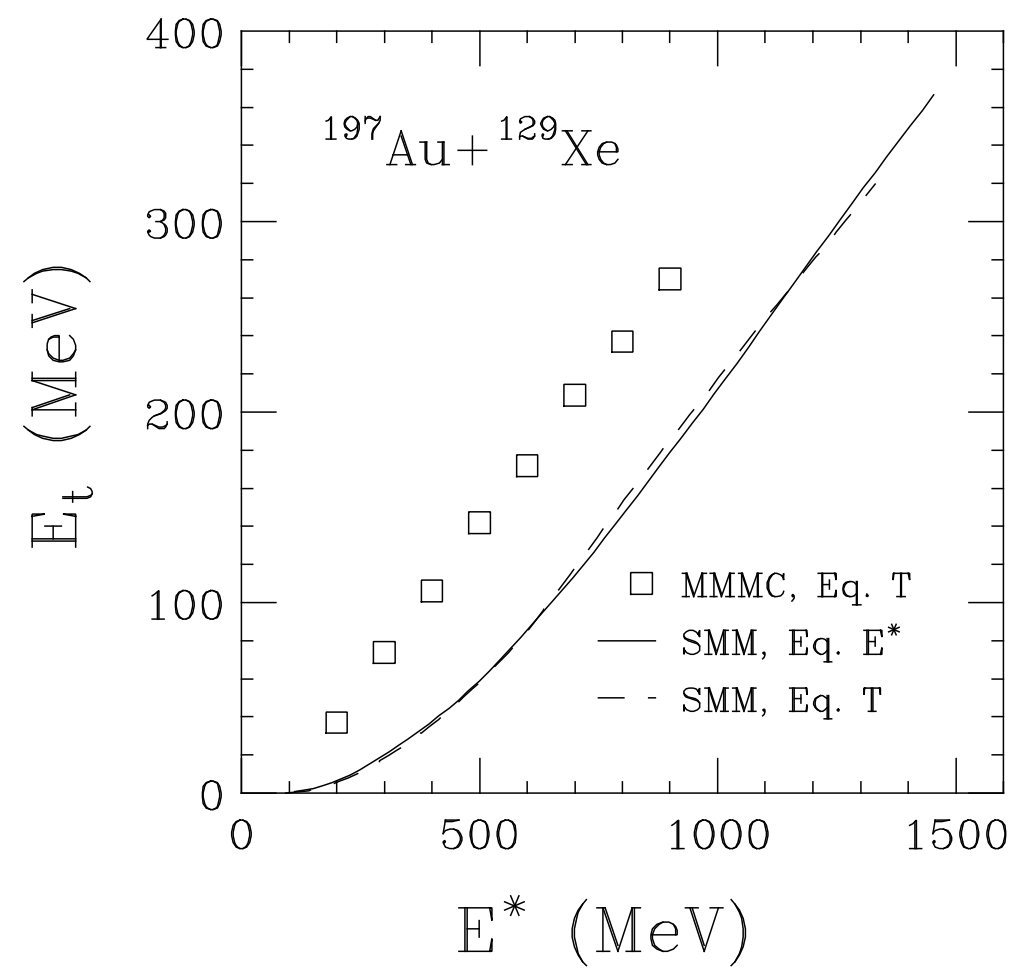

Fig. 2. Dependence of the transverse energy $E_{t}$ on the total excitation energy $E^{*}$ for the reaction ${ }^{197} \mathrm{Au}+{ }^{129} \mathrm{Xe}$, as predicted by the $\mathrm{SMM}^{7}$ (lines), GEMINI ${ }^{20}$ (circles), and MMMC ${ }^{8}$ (boxes) codes. The solid and dashed lines represent calculations made assuming equal excitation energies $E^{*}$ or equal temperatures $T$ of the projectile- and target-like fragments.

Fig. 2 shows the average functional dependence of $E_{t}$ on $E^{*}$, as predicted by the codes $\mathrm{SMM}$ (solid and dashed lines) and GEMINI29 (circles) for the system ${ }^{197} \mathrm{Au}+{ }^{129} \mathrm{Xe}$ discussed in a recent paper. 1 This system is very close to the ${ }^{209} \mathrm{Bi}+{ }^{136} \mathrm{Xe}$ system, discussed earlier above, for which the joint multiplicity of neutrons and charged particles has been actually measured. Hence, it is expected that the SMM and GEMINI models provide for an adequate description of this system as well and, in particular, for the description of the initial segment of the $m_{n}-m_{L C P}$ correlation ridge parallel to the $m_{n}$ axis. It is the presence of this segment that translates directly and unambiguously into the strong nonlinearity of the functional relationship between $E_{t}$ and $E^{*}$ and the lack of a direct proportionality between these two quantities in the range of excitation energies considered in the above papers. 10 . 24 For 
comparison, the predictions by the code MMMCl are shown in Fig. 2 by squares. As expected based on the trends exhibited by the MMMC calculations in Fig. 1, the MMMC predicts almost a straight proportionality between $E_{t}$ and $E^{*}$.

The two almost overlapping curves in Fig. 2 represent two extreme assumptions made regarding the excitation energy division between Au-like and Xe-like primary fragments, equal excitation energies or equal temperatures (excitation energies are proportional to the masses) of projectile-like and target-like fragments. Obviously, the results exhibit a remarkable insensitivity to the excitation energy division between the fragments. The non-linear functional dependence seen in Fig. 2 can be approximated by two linear segments: $E_{t}=0$, for $E^{*}<E_{o}^{*}$, and $E^{*}=a\left(E^{*}-E_{o}^{*}\right)$ for $E^{*}>E_{o}^{*}$, where the offset is $E_{o}^{*} \approx 340 \mathrm{MeV}$ and the slope is $a \approx 0.25$.

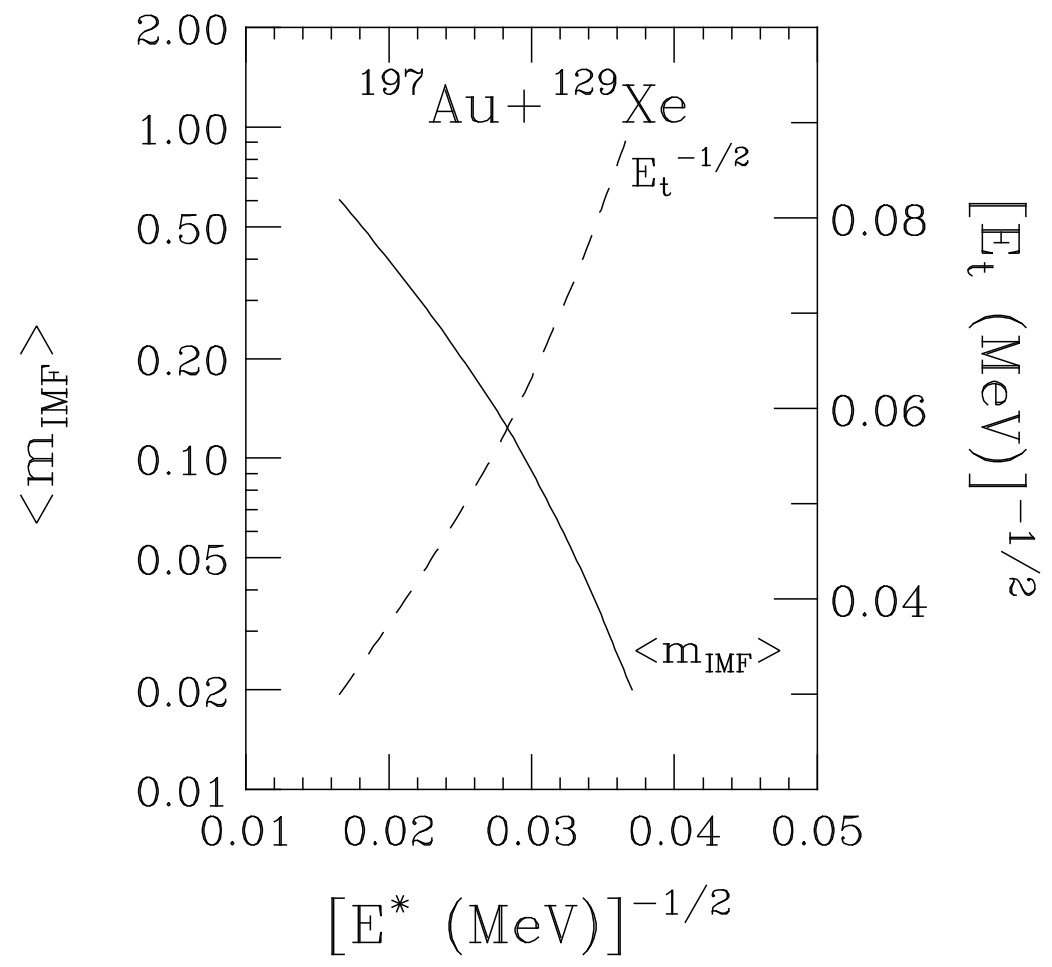

Fig. 3. Nonlinearity of the relationship between the inverse square-roots of $E_{t}$ and $E^{*}$ (dashed line) and the resulting nonlinearity of an Arrhenius-like plot, obtained by converting the abscissa of a previously published ${ }^{10,11}$ pseudo-Arrhenius plot for the system ${ }^{197} \mathrm{Au}+{ }^{129} \mathrm{Xe}$ (solid line). 
The functional dependence seen in Fig. 2 results in a strongly nonlinear relationship between the true "thermal" Arrhenius variable $1 / T$ or its measure $1 / \sqrt{E}^{*}$, on the one hand, and the pseudo-Arrhenius variable $1 / \sqrt{E_{t}}$, on the other hand, over the full range of values considered in the recent papers. 13 造 This relationship is illustrated by the dashed line in Fig. 3. Accordingly, the persistent 10.11 striking linearity of experimental pseudo-Arrhenius plots implies a similarly persistent nonlinearity of such plots when converted to a true Arrhenius abscissa $1 / T$ or $1 / \sqrt{E^{*}}$. To further demonstrate this point, the solid line in Fig. 3 represents a typical experimental plot converted to a representation where $1 / \sqrt{E^{*}}$ is the abscissa variable. The solid line is obtained based on experimental data 11 for a representative atomic number of $Z=8$ and the relationship between $E_{t}$ and $E^{*}$ seen in Fig. 2. Obviously, the nonlinearity of the Arrhenius-like plot in Fig. 3 contradict previous conclusions of thermal10 11 or microcanonical12.13 scaling of IMF distributions. These observations also demonstrate independently that true and pseudo-Arrhenius plots are not equivalent to each other, a fact that has been pointed out earlier $9[44$.

In conclusion, the joint distributions of neutron and charged-particle multiplicities offer a powerful tool for scrutinizing certain theoretical models and concepts. This has been demonstrated here for two different examples. While the statistical-equilibrium model GEMINI20 and the Copenhagen model of simultaneous multifragmentation $\mathrm{SMM}$ - provide a quantitative account of these distributions, the Berlin model of microcanonical fragmentation does not predict the characteristic long $m_{L C P} \approx 0$ segment in the $m_{n}$ vs. $m_{L C P}$ correlation ridge. The experimental joint $m_{n}$ vs. $m_{L C P}$ multiplicity distributions confirm predictions by phase-space models that there is an appreciable offset (in $E^{*}$ ) in the approximately linear functional relationship between average transverse energy $E_{t}$ of charged particles and average total excitation energy $E^{*}$ at energies above some threshold energy of $E_{o}^{*} \approx 340 \mathrm{MeV}$. This observation challenges conclusions regarding thermal10.11.24 or microcanonical 12.13 scaling of multifragmentation, which are all based on the assumption of a direct proportionality between $E_{t}$ and $E^{*}$. The present study has demonstrated again the difficulty to interpret in an unambiguous fashion isolated effects observed in complex nuclear reactions. This expe- 
rience stresses the necessity of a more holistic analysis of energetic nuclear reactions, where many facets of experimental observations are considered simultaneously. Such an approach requires the simultaneous measurement of as many experimental observables as possible and singling out all "probative" correlations that contradict some but not other theoretical or intuitive concepts. More specifically, the present work has demonstrated the great importance of measuring neutron data along with those for charged reaction products.

This work was supported by the U.S. Department of Energy grant No. DE-FG0288ER40414. 


\section{REFERENCES}

1. C.K. Gelbke and D.H. Boal, Prog. Part. Nucl. Phys. 19, 33 (1987), and references therein.

2. B. Borderie, M.F. Rivet, and L. Tassan-Got, Ann. Phys. (Fr) Vol.15 (1990), and ref. therein.

3. L.G. Moretto and G.J. Wozniak, Annu. Rev. Nucl. Part. Sci. 43379 (1993), and references therein

4. G.F. Bertsch et al., Phys. Rep. 160, 189 (1988)

5. W.A. Friedman, Phys. Rev. Lett. 60, 2125 (1988); W.A. Friedman, Phys. Rev. C42, $667(1990)$

6. J. Aichelin, Phys. Rep. 202, 233 (1991).

7. J.P. Bondorf et al., Phys. Rep. 257, 133 (1995).

8. D.H.E. Gross, Rep. Prog. Phys. 53, 605 (1990).

9. J. Tõke et al., Phys. Rev. Lett. 77, 3514 (1996).

10. L. G. Moretto et al. Phys. Rev. Lett. 74, 1530 (1995); K. Tso et al. Phys. Lett. B361, 25 (1995); L.G. Moretto, R. Ghetti, L. Phair, K. Tso, and G.J. Wozniak, Phys. Rep. 287, 249 (1997).

11. L. Beaulieu, L. Phair, L.G. Moretto, and G.J. Wozniak, Phys. Rev. Lett. 81, 770 (1998).

12. A.S. Botvina and D.H.E. Gross, Phys. Lett. B344, 6 (1995).

13. A.S. Botvina and D.H.E. Gross, Phys. Rev. C58, R23, (1998).

14. B. Tsang and P. Danielewicz, Phys. Rev. Lett. 81, 770 (1998).

15. W. Skulski, et al., in print in Phys. Rev. C., April 1999. 
16. W.U. Schröder, Nucl. Phys.A538, 439c (1992).

17. J. Tõke, et al. Phys. Rev. Lett. 75, 2920 (1995).

18. D.X.Jiang et al., Nucl. Phys.A503, 560 (1989).

19. X. Ledoux et al., Phys. Rev. C57, 2375 (1998); F. Goldenbaum et al., Phys. Rev. Lett. 77, 1230 (1996).

20. R.J. Charity, computer code GEMINI, available via an anonymous ftp from wunmr.wustl.edu/pub/gemini.

21. S.P. Baldwin, Ph.D. thesis. University of Rochester, Rochester, New York, 1994.

22. J. Poitou and C. Signarbieux, Nucl. Instr. Meth. 114, 113 (1974).

23. B.M. Quednau, S.P. Baldwin, M.B. Chatterjee, J.R. Huizenga, W.U. Schröder, B.M. Szabo, J. Tõke, U. Jahnke, D. Hilscher, H. Rossner, B. Lott, S. Bresson, J. Galin, D. Guerreau, M. Morjean, and D. Jacquet, Phys. Lett. B309, 10 (1993).

24. L.G. Moretto, L. Phair, and G.J. Wozniak, Phys. Rev. C60, R031601 (1999). 